\title{
Mathematical Model of Pulp Washing Using Mathematica
}

\author{
Jitender Kumar ${ }^{1, a}$, V.K. Kukreja ${ }^{2}$ \\ ${ }^{1}$ Department of Mathematics, BGIET, Sangrur, 148001 (Punjab) INDIA \\ ${ }^{2}$ Department of Mathematics, SLIET, Longowal, 148106 (Punjab) INDIA
}

\begin{abstract}
Good washing is the key to a successful closure of the water system in a pulp production line. An improved understanding of associated phenomena, particularly those related to the removal of the very last impurities, is therefore desirable. Therefore a mathematical model describing the desorption phenomenon. The system of governing partial differential equations is solved using Mathematica. Model is simulated using the industrial data. The output thus obtained is compared with the analytic and numerical results of earlier workers. Present technique is simple, elegant and convenient for solving two point boundary value problems with varying range of parameters.
\end{abstract}

Keywords: Peclet number, diffusion-dispersion, Mathematica

\section{Introduction}

An integral part of chemical recovery is the washing process. Methods of describing the efficiency of the various washing operations are necessary before an analysis of the overall consequences of recycling or mill closure is possible. Washing is simply bulk removal of the liquor surrounding the pulp fibers. In a laboratory washing cell, the mechanism of the displacement washing of unbleached Kraft pulp was investigated. The efficiency of displacement washing depends on the degree of mixing and also on the rate of desorption and diffusion of dissolved solids and chemicals from the pulp fibers. The separation of pulp and paper industry to produce its target production with high efficiency and less environmental load can only be met by initiating a meticulously planned research on mathematical methods. Modeling of pulp washing is done mainly using three approaches namely (a) Process modeling (b) Physical modeling (c) Statistical modeling. A complete review of the various process models used to describe pulp washing has been presented by Pekkanen and Norden [9]. Initially researchers Brenner [1] and Pellet [8] has introduced a mathematical model combining the effects of particle diffusion and axial dispersion. An extensive model related to mass transfer in fibrous particle was given by Grahs [2], it was also restricted for axial dispersion only. Moreover certain physical features of the fibers such as fiber porosity and fiber radius were ignored. Lapidus and Amundson [7] also followed two adsorption isotherms; one implies that equilibrium is established at each point in the bed while the other assumes the rate of adsorption is finite.

Washing models of pulp based on axial dispersion and particle diffusion along with adsorption isotherms for sodium and lignin are fairly established and also solved by earlier workers such as Arora et al. (2006, 2009), Babu et al. (2004), Brenner (1962), Kukreja (1996, 2009), Kumar (2002), Kumar et al. (2009), Potucek et al. (2006), Sotelo et al. (2004), Sridhar et al. (1999). Kukreja (1996) solved the washing model analytically by using Laplace transform technique and Kumar (2002) tried to solve numerically using finite difference technique.

The accuracy of the analytic solution undoubtedly exceeds the limit of applicability of the theory to real situations. Moreover, it is highly desirable to have a simple and consistent model of the transport phenomenon based on essential features of real situation. Keeping this modest goal in mind, the method of separation of variables is first applied on partial differential equation and then Laplace transform is taken of the equations.

\section{Model based on axial dispersion}

The displacement washing model based on the axial dispersion and particle diffusion describing the washing zone is given by

$$
\frac{\partial c}{\partial t}+u \frac{\partial c}{\partial z}+\mu \frac{\partial n}{\partial t}=D_{L} \frac{\partial^{2} c}{\partial z^{2}}
$$

\footnotetext{
a Corresponding author: jitenderrattan2005@gmail.com
} 
with adsorption isotherm

$$
n=k c \text {. }
$$

This equation represent the basis for the mathematical models of displacement washing, where $t$ is the time from the commencement of the displacement, $z$ is the distance from the point of introduction of the displacing fluid, $c=c(z, t)$ is the solute concentration, $D_{L}$ is longitudinal dispersion coefficient, $u$ is the average interstitial velocity of the fluid and $L$ is thickness of the packed bed.

On account of unusual nature of displacement process, appropriate boundary conditions have been extensively discussed in the literature (Brenner, 1962; Shelly et al., 2006; Shirashi, 2001; Szukiewicz, 2000) like Danckwerts' conditions at the inlet and outlet of the bed:

$$
\begin{array}{ll}
c=c_{s} & \text { at } z=0, \\
\frac{\partial c}{\partial z}=0 & \text { at } z=L .
\end{array}
$$

Accordingly, initial condition is given by

$$
c(z, 0)=n(z, 0)=c_{i} \text { for } 0<t<\frac{L}{u} .
$$

\subsection{Conversion of model in to dimensionless form:}

Equations (1) to (5) can be put in dimensionless form using dimensionless variables:

$$
\begin{array}{r}
C=\frac{c-c_{s}}{c_{i}-c_{s}} ; N=\frac{n-c_{s}}{c_{i}-c_{s}} \\
Z=\frac{z}{L} ; T=\frac{u t}{(1+\mu k) L}
\end{array}
$$

The dimensionless time, $T$, corresponds physically to the number of pore displacements introduced into the medium since the starts of the experiment; that is, it is equal to the ratio of total fluid volume introduced to the free value of the bed.

By these means, equation (1) becomes

$$
\frac{\partial C}{\partial T}+\frac{\partial C}{\partial Z}=\frac{1}{P e} \frac{\partial^{2} C}{\partial Z^{2}}
$$

where $P e=\frac{u L}{D_{L}}$ is the Peclet number. The boundary

conditions are now of the form

$$
\begin{aligned}
& C(0, T)=0 \text { at } X=0 \text { for } T>0 \\
& \frac{\partial C(1, T)}{\partial Z}=0 \quad \text { at } X=1 \text { for } T>0
\end{aligned}
$$

While the initial condition is

$$
C=1 \text { at } T=0 \text { for } 0<Z \leq 1
$$

Now, our main aim is to estimate $C=C(Z, T)$ satisfying Eqs. (7)-(9), which will eventually lead to exit solute concentration $C_{e}=C_{e}(T)=C(1, T)$.

\section{Solution of model}

Method of separation of variables is used for solving Eq. (6). This method transforms the partial differential equation into a system of ordinary differential equation, each of which depends only on one of the functions and the solution is given as product of the functions.

Finally, the solute concentration at any location and time in the bed can be written as:

$C(Z, T)=\sum_{n=1}^{\infty} \frac{\int_{0}^{1} e^{-\frac{P e Z}{2}} \sin \left(\alpha_{n} Z\right) d Z}{\int_{0}^{1} \sin ^{2}\left(\alpha_{n} Z\right) d Z} \exp \left(\frac{P e Z}{2}-\frac{P_{n}^{2} T}{P e}\right) \sin \alpha_{n} Z$

where the $\alpha_{n}(n=1,2,3, \ldots)$ are the positive roots, taken in order of increasing magnitude, of the transcendental equation

$\tan \alpha=-\frac{2 \alpha}{P e}$ and $P_{n}=\sqrt{\frac{4 \alpha_{n}{ }^{2}+P e^{2}}{4}}$

The concentration of solute in the dimensionless form for any location and time can be found by Laplace transform as Kukreja [3].

$$
\frac{c-C_{s}}{C_{i}-C_{s}}=\sum_{n=1}^{\infty} \frac{4 \beta_{n}^{2} e^{P_{n} T} e^{\frac{P_{e} Z}{2}}\left[P_{e} \sin \left\{(Z-1) \beta_{n}\right\}-2 \beta_{n} \cos \left\{(Z-1) \beta_{n}\right\}\right]}{P_{e} P_{n} \sin \beta_{n}\left(P_{e}^{2}+4 \beta_{n}^{2}+2 P_{e}\right)}
$$

(9)

The concentration of solute in the dimensionless form for any location and time can be found by separation of variables as Grahs (1974).

$$
C(x, T)=\exp \left\{\frac{B_{0}}{2}\left(x-\frac{T}{2}\right)\right\} \cdot \sum_{n=1}^{\infty} \frac{4 w_{n}^{2} \sin w_{n} x \cdot \exp \left(-\frac{1}{B_{0}} w_{n}^{2} T\right)}{\left(2 w_{n}-\sin 2 w_{n}\right)\left(\frac{B_{0}}{4}+w_{n}^{2}\right)}
$$

Where $w_{n}$ are the positive roots to the equation

$$
\tan w_{n}=-\frac{4 w_{n}}{B_{0}}
$$

\section{Results and Discussion}

The dimensionless model equation (7) of longitudinal mixing is solved for $\mathrm{Pe}=1.1$ with Mathematica. The behavior of the dimensionless concentration with respect to the dimensionless time and dimensionless distance is shown in the Figure-1. The results at dimensionless distance at $\mathrm{X}=1$ are compared with work of previous researchers as shown in the figure 2. Brenner [1] The mathematical model developed through transport phenomena had solved the equation (7) (dimensionless form) analytically, while Grahs [2] had used orthogonal Comparison shows that Mathematica gives good accuracy and the magnitude of error is very low. It can be seen that at $\mathrm{T}=0.0$ the error is $0.001 \%$ which is minimum and oscillate up to $6.50 \%$ in such a way 
that at $\mathrm{T}=0.1$ error is $0.5 \%$, at $\mathrm{T}=0.2$ error is $6.50 \%$, at $\mathrm{T}=0.3$ error is $5.72 \%$, at $\mathrm{T}=0.4$ error is $4.56 \%$, at $\mathrm{T}=0.5$ error is $4.56 \%$, at $\mathrm{T}=0.6$ error is $3.48 \%$, at $\mathrm{T}=0.7$ error is $2.54 \%$, at $\mathrm{T}=0.8$ error is $1.76 \%$, at $\mathrm{T}=0.9$ error is $.57 \%$, at $\mathrm{T}=1.0$ error is $0.13 \%$, at $\mathrm{T}=1.1$ error is $0.22 \%$, at $\mathrm{T}=1.2$ error is $0.50 \%$. Since the error is increasing up to time $\mathrm{T}=0.2$ and after that decrease at time $\mathrm{T}=1.0$ and for above at time $\mathrm{T}=1.0$ again shows an increasing trend. Solutions from all the techniques analytic as well as numerical used by Brenner [1], Grahs [2] and "Mathematica" solver shows fluctuations in the results when time is increased above $\mathrm{T}=1.2$.

Hence comparison of the results for significant accuracy has been carried out up to time $\mathrm{T}=1.2$. However one very interesting observation is that the orthogonal collocation method used by Grahs [2] gives an increasing trend from $\mathrm{T}=0.5$ to $\mathrm{T}=0.6$ and thereafter amplitude starts decreasing consistently. This is contrary to analytical solution of Brenner [1] which shows a continuous decreasing trend from 1.0 to .0854 . In the present investigation we also obtain the decreasing trend consistently as depicted in the analytical solution by Brenner [1].

The results at dimensionless distance $X=1$, are obtained by using 22 meshes in both dimensionless time and dimensionless distance $\mathrm{x}$ are given in table-1. The results are also compared with results obtained by Kumar [4] using finite difference method. In this case, also results as obtained by Brenner [1] and numerical results by Grahs [2] and show better accuracy than Kumar [4].

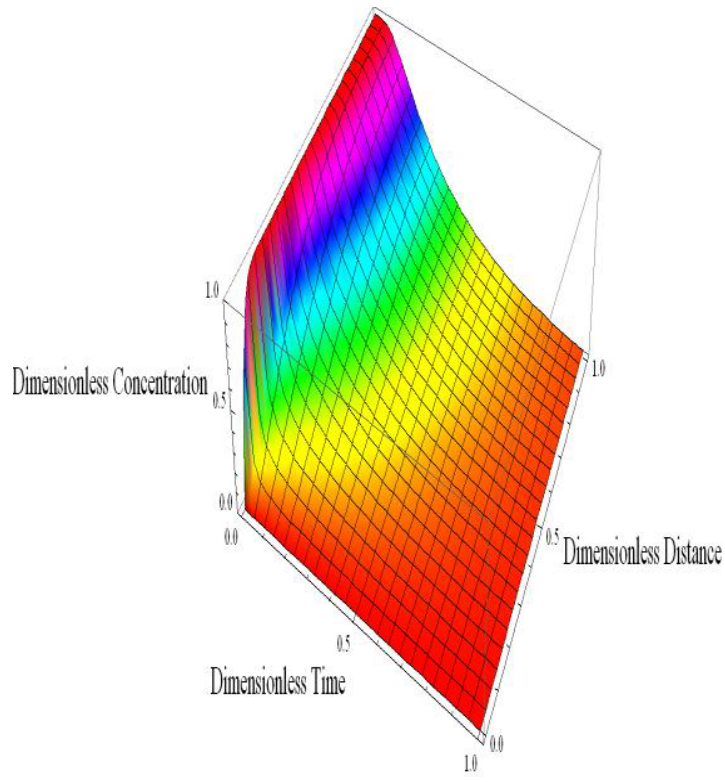

Figure 1. Solution of the Model equation (7) by Mathematica

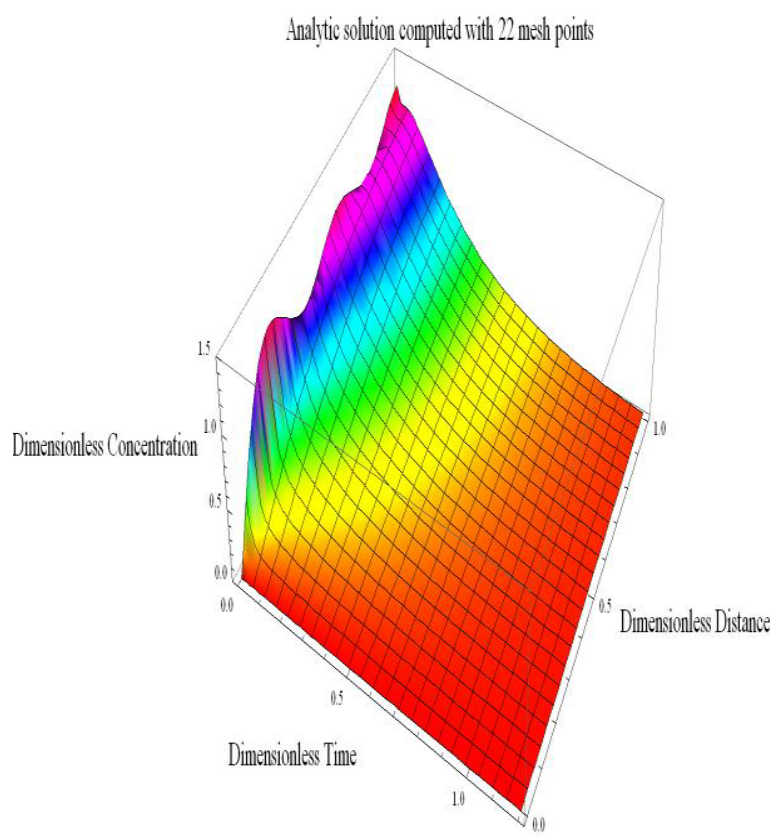

Figure 2. Solution of the Model equation (7) by Grahs (1974)

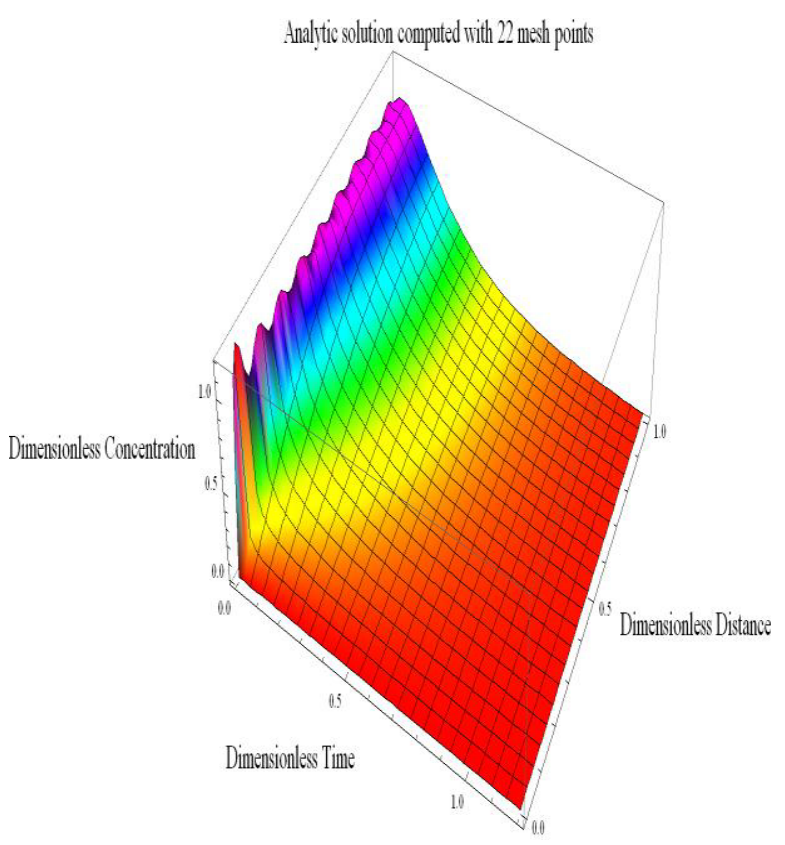

Figure 3. Solution of the Model equation (7) by Kukreja (1996) 


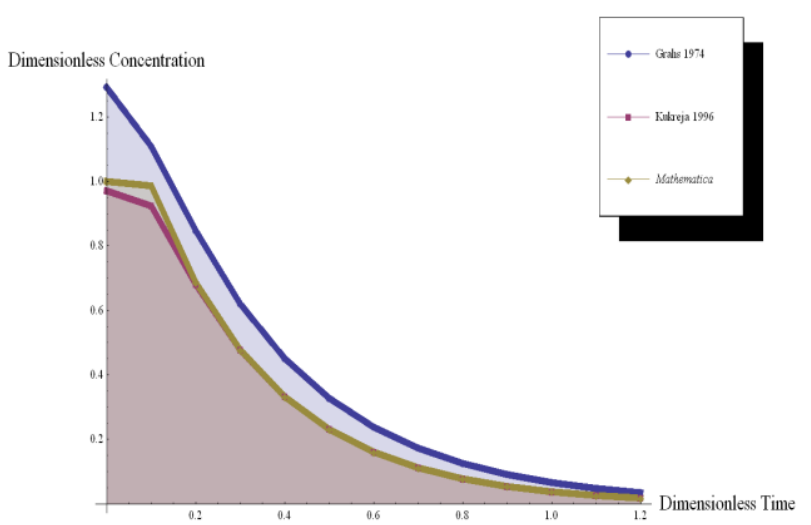

Figure 4. Comparison of Analytical solution of Brenner with Present investigation by Mathematica at Dimensionless distance $\mathrm{X}=1$ at same $P_{e}=1.1$

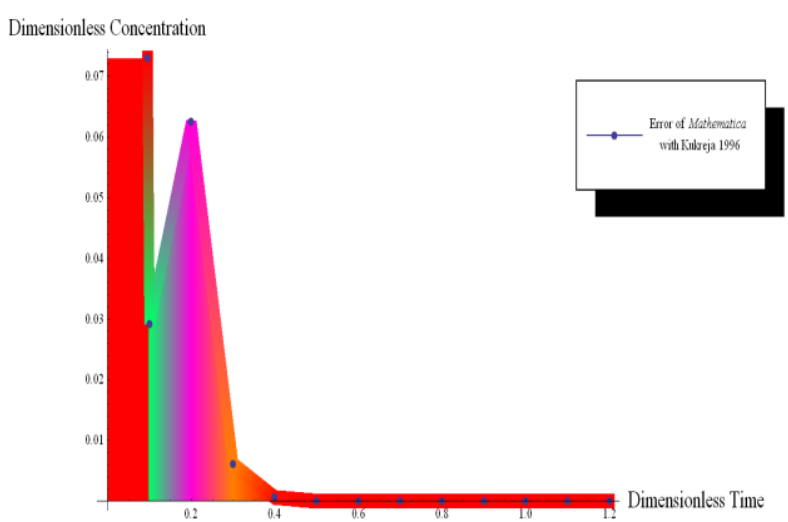

Figure 5. Error of Mathematica with Kukreja (1996)

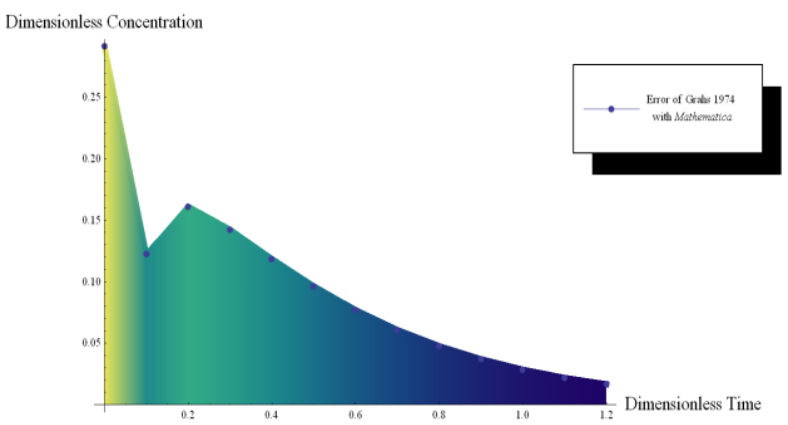

Figure 6. Error of Mathematica with Graph
Table1 Comparison of Analytical solution of Grahs (1974) and Kukreja (1996) with Present investigation by Mathematica at same $P e=1.1$

\begin{tabular}{|c|c|c|c|}
\hline $\begin{array}{c}\text { Time } \\
\mathrm{T}\end{array}$ & $\begin{array}{c}\text { Grahs(1974) } \\
\text { Analytical } \\
\text { Solution } \\
P e=1.1\end{array}$ & $\begin{array}{l}\text { Kukreja } \\
(1996) \\
\text { Analytical } \\
\text { Solution } \\
P e=1.1\end{array}$ & $\begin{array}{c}\text { Analytical } \\
\text { Solution } \\
\text { By } \\
\text { Mathematica } \\
P e=1.1\end{array}$ \\
\hline 0.00 & 1.2913 & 0.9708 & 1.0000 \\
\hline 0.10 & 0.1087 & 0.9239 & 0.9864 \\
\hline 0.20 & 0.8477 & 0.6805 & 0.6866 \\
\hline 0.30 & 0.6203 & 0.4773 & 0.4779 \\
\hline 0.40 & 0.4512 & 0.3326 & 0.3326 \\
\hline 0.50 & 0.3279 & 0.2315 & 0.2315 \\
\hline 0.60 & 0.2382 & 0.1611 & 0.1611 \\
\hline 0.70 & 0.1731 & 0.1122 & 0.1122 \\
\hline 0.80 & 0.1258 & 0.0781 & 0.0781 \\
\hline 0.90 & 0.0914 & 0.0543 & 0.0543 \\
\hline 1.00 & 0.0664 & 0.0378 & 0.0378 \\
\hline 1.10 & 0.0482 & 0.0263 & 0.0263 \\
\hline 1.20 & 0.0350 & 0.0183 & 0.0183 \\
\hline
\end{tabular}

\section{Conclusion}

As the model so developed is mathematically complex to solve, so this is simplified for further investigation in order to compare some of the models proposed by Grahs [2] and Brenner [1] and Kumar [4] but with different numerical technique. The model with varying initial and boundary conditions is proposed to solve through Mathematica. The present technique is validated with the solutions of Grahs [2] based on numerical technique (Orthogonal Collocation) and Brenner [1] with analytical methods using Kumar [4]. Hence it may be concluded that the Mathematica used in 
the present investigation is simple, elegant and convenient for solving two point boundary value problems and average numerical errors. The algorithms in this solver are easy to set up, and so the method represents an advantage and good alternate to the available techniques for such type of problems.

\section{Nomenclature}

$A_{c}$ : Area of cross section of washing zone, $\mathrm{m}^{2}$

c: Concentration of the solute in the liquor, $\mathrm{kg} / \mathrm{m}^{3}$

$\mathrm{C}$ : Dimensionless solute concentration

DL: Longitudinal dispersion coefficient, $\mathrm{m}^{2} / \mathrm{s}$

Pe : Peclet number $\left(=\mathrm{uL} / \mathrm{DL}_{\mathrm{L}}\right) \mathrm{G}, \mathrm{H} \quad$ : Constants

$\mathrm{T}$ : Dimensionless time

$\mathrm{X}$ : Dimensionless distance

$\mathrm{u}$ : Liquor speed in cake pores, $\mathrm{m} / \mathrm{s}$

\section{References}

1. Brenner H., "The diffusion model of longitudinal mixing in beds of finite length, Numerical values", Chemical Engineering Science, 17 , pp.229-243, (1962)

2. Grah L.E., "Displacement washing of packed beds of cellulose fibers, part-1: Mathematical model,

3. Kukreja V. K., "Modeling of washing of brown stock on rotary vacuum washer ", $\mathrm{PhD}$. Thesis, University of Roorkee, Roorkee, India, (1996)

4. Kumar Mukesh, "Mathematical modeling of pulp washing systems and solutions", $\mathrm{PhD}$. Thesis, Indian institute of Technology Roorkee, Roorkee, India, (2002)

5. Kumar Deepak, Kumar Vivek, Singh V.P, "Numerical Solution of Brown Stock Washer

Problems in Paper Industry", $11^{\text {th }}$ Punjab Science Congress, Thapar University Patiala, India, Feb. 7-9, (2008)

6. Lee P.F., "Optimizing the displacement washing of pads of wood pulp fibres", Tappi J., 62 (9), pp.7578, (1979)

7. Lapidus L. and Amundson N.R., "Mathematics of Adsorption in beds, part-vi: The effect of longitudinal diffusion in ion exchange and chromatographic columns", J. of Physical Chemistry, 56, pp.984-988, (1952)

8. Pellett G. L., " Longitudinal dispersion, intra particle diffusion and liquid-phase mass transfer during flow through multi particle systems", Tappi J., 49(2), pp.75-82, (1966)

9. Pekkanen M. and Norden H. V., "Review of pulp washing models", Paperija Puu, 67(11), pp.689-696, (1985) 